\title{
Muscle Expression of SOD1 1 G93A Modulates microRNA and mRNA Transcription Pattern Associated with the Myelination Process in the Spinal Cord of Transgenic Mice
}

\section{OPEN ACCESS}

Edited by:

Manoj Kumar Jaiswal,

Columbia University Medical Center,

USA

Reviewed by:

Dumitru A. lacobas, Albert Einstein College of Medicine of

Yeshiva University, USA

Deborah Baro,

Georgia State University, USA

Eva R. Chin,

University of Maryland School of

Public Health, USA

*Correspondence:

Antonio Musarò

antonio.musaro@uniroma1.it

${ }^{\dagger}$ These authors have contributed equally to this work.

Received: 09 June 2015 Accepted: 13 November 2015 Published: 01 December 2015

Citation:

Dobrowolny G, Bernardini C, Martini M, Baranzini M, Barba M and Musarò A (2015) Muscle Expression of SOD 1 G93A Modulates microRNA

and mRNA Transcription Pattern Associated with the Myelination

Process in the Spinal Cord of Transgenic Mice.

Front. Cell. Neurosci. 9:463. doi: 10.3389/fncel.2015.00463

\section{Gabriella Dobrowolny ${ }^{1,2 \dagger}$, Camilla Bernardini ${ }^{3 \dagger}$, Martina Martini ${ }^{1,2}$, Mirko Baranzini $^{3}$, Marta Barba ${ }^{3}$ and Antonio Musarò ${ }^{1,2 *}$}

1 DAHFMO-Unit of Histology and Medical Embryology, Institute Pasteur-Cenci Bolognetti, IIM, Sapienza University of Rome, Rome, Italy, ${ }^{2}$ Center for Life Nano Science at Sapienza, Istituto Italiano di Tecnologia, Rome, Italy, ${ }^{3}$ Institute of Anatomy and Cell Biology, Università Cattolica del Sacro Cuore, Rome, Italy

A crucial system severely affected in several neuromuscular diseases is the loss of effective connection between muscle and nerve, leading to a pathological non-communication between the two tissues. One of the best examples of impaired interplay between muscle and nerve is Amyotrophic Lateral Sclerosis, a neurodegenerative disease characterized by degeneration of motor neurons and muscle atrophy. Increasing evidences suggest that damage to motor neurons is enhanced by alterations in the neighboring non-neuronal cells and indicate that altered skeletal muscle might be the source of signals that impinge motor neuron activity and survival. Here we investigated whether muscle selective expression of SOD1 ${ }^{\mathrm{G} 93 \mathrm{~A}}$ mutant gene modulates mRNAs and miRNAs expression at the level of spinal cord of MLC/SOD1G93A mice. Using a Taqman array, the Affymetrix Mouse Gene 2.0 ST approach and the MiRwalk 2.0 database, which provides information on miRNA and their predicted target genes, we revealed that muscle specific expression of SOD1 ${ }^{\mathrm{G} 93 \mathrm{~A}}$ modulates relevant molecules of the genetic and epigenetic circuitry of myelin homeostasis in spinal cord of transgenic mice. Our study provides insights into the pathophysiological interplay between muscle and nerve and supports the hypothesis that muscle is a source of signals that can either positively or negatively affect the nervous system.

Keywords: muscle-nerve interplay, miRNA and mRNA signature, ALS, myelination process, SOD1G93A

Abbreviations: ALS, Amyotrophic Lateral Sclerosis; ANOVA, ANalysis Of VAriance; Cldn19, Claudin 19; DAVID, Database for Annotation Visualization Integrated Discovery; Dhh, Desert hedgehog; DIANA, Dna Intelligence ANAlysis; dmax, maximum axonal diameter; Dmax, maximum fiber diameter; Dmin, minimum fiber diameter; dmin, minimum axonal diameter; Drp2, Dystrophin related protein 2; Egr2, Early growth response 2; FC, Fold Change; FVB, Friend leukemia virus B strain; GEO database, Gene Expression Omnibus database; Gldn, Gliomedin; GO, Gene Ontology; KEGG, Kyoto Encyclopedia of Genes and Genomes; Mag, Myelin-associated glycoprotein; Matn2, Matrilin 2; mIGF-1, muscle specific isoform of the Insulin Growth Factor-1; miRNA, miR, microRNA; miRPath, miRNA pathway; MLC, Myosin Light Chain; Mpz, Myelin protein zero; Nkx2-2, NK2 homeobox 2; Ogn, Osteoglycin; OMIM, Online Mendelian Inheritance in Man; Pmp22, Peripheral myelin protein 22; Prx, Periaxin; SDS, Sodium Dodecyl Sulfate; Smtn, Smoothelin; SOD1, Superoxide dismutase 1; STRING, Search Tool for the Retrieval of Interacting Genes/Proteins; TAC, Transcriptome Analysis Console; UTR, Untranslated region. 


\section{INTRODUCTION}

Amyotrophic Lateral Sclerosis (ALS) is a fatal neurodegenerative disorder in which the functional connection between nerve and muscle is severely compromised (Musarò, 2013). ALS affects pyramidal neurons in the motor cortex and lower motor neurons originating in the brainstem and in the spinal cord. The most typical feature of this progressive lethal disease is the degeneration of motor neurons, muscle weakness and atrophy, that leads to progressive paralysis and death by respiratory failure in few years (Musarò, 2010, 2013). ALS is epidemiologically classified into two major forms: sporadic (90-95\%) and familial (5-10\%) form. Among the familial cases, $\sim 20 \%$ are caused by dominantly inherited mutations in the $\mathrm{Cu} / \mathrm{Zn}$ superoxide dismutase (SOD1) protein (Rosen, 1993) a cell scavenger of superoxide.

One of the critical issue that remains to be addressed in ALS is whether motor neurons are the first and sole direct targets of ALS or if the toxic activity of mutant SOD1 is cell autonomous. Recent experimental evidence revealed that ALS is a multi-factorial and multi-systemic disease in which alterations in structural, physiological, and metabolic parameters in different cell types, such as motor neurons, glia, and muscle cells, may act synergistically to exacerbate the disease (Boillée et al., 2006; Julien, 2007; Musarò, 2013). Skeletal muscle is a source of signals that influence neuron survival, axonal growth and maintenance of synaptic connections. Notably, development in the absence of skeletal muscle results in the sequential ablation of motor neurons from the spinal cord to the brain (Kablar and Rudnicki, 1999); thus, effective connection between muscle and nerve is crucial for the survival and function of both tissues throughout life. We have previously demonstrated that muscle-specific expression of SOD1 mutation in the MLC/SOD $1{ }^{\mathrm{G} 93 \mathrm{~A}}$ transgenic mouse model, which selectively expresses the mutant SOD 1 G93A gene in the skeletal muscle under the transcriptional control of a muscle-specific promoter (MLC), induces accumulation of Reactive Oxygen Species, causes muscle atrophy with a concomitant alteration in the ultrastructure and in the functional performance of skeletal muscles, and promotes microglia activation in the spinal cord, that is a presymptomatic sign of ALS disease (Dobrowolny et al., 2008b). Moreover, it has been reported that skeletal muscle-restricted expression of the human mutant SOD1 gene causes motor neuron degeneration in old transgenic mice (Wong and Martin, 2010) and that muscle-selective alterations in mitochondrial function initiate neuromuscular junction alteration, distal axonopathy, astrocytosis, and mild motor neuron loss (Dupuis et al., 2009). We also demonstrated that forced expression of muscle specific isoform of the Insulin Growth Factor-1 (mIGF-1) exclusively in the skeletal muscle of the global SOD1 ${ }^{\mathrm{G} 93 \mathrm{~A}}$ mice counteracted the symptoms of ALS, induced satellite cell activation, stabilized neuromuscular junctions, preserved motor peripheral nerve, and led to a reduction in astrocytosis in the SOD $1^{\mathrm{G} 93 \mathrm{~A}}$ spinal cord (Dobrowolny et al., 2005, 2008a). All together these data indicate that nervous development and homeostasis are intimately coupled to skeletal myogenesis and muscle function. Moreover, these findings support the hypothesis that skeletal muscle is a primary target of mutant SOD1 toxicity in mice and indicate that altered skeletal muscle impairs motor neuron activity, suggesting a sequential pattern of degeneration in which muscle abnormalities precede motor neuron death. In order to add new insights into the pathogenesis of ALS and define whether skeletal muscle alteration potentially affect the nervous system we analyzed the mRNA and miRNA expression in the lumbar ventral spinal cord of $\mathrm{MLC} / \mathrm{SOD} 1^{\mathrm{G} 93 \mathrm{~A}}$ transgenic mouse model (Dobrowolny et al., 2008b). Interestingly, we found that muscle specific expression of SOD ${ }^{\mathrm{G} 93 \mathrm{~A}}$ gene affects spinal cord miRNome and transcriptome; in particular we observed the activation of genes involved in the myelination process and a decrease in the axon diameter to total fiber diameter in the sciatic nerve, suggesting a myelinopathy in the transgenic mouse model.

\section{MATERIALS AND METHODS}

\section{Mice}

Animal model used: 4 month old MLC/SOD $1{ }^{\mathrm{G} 93 \mathrm{~A}}$ mice overexpressing the mutant $\mathrm{SOD} 1$ gene $\left(\mathrm{SOD} 1^{\mathrm{G} 93 \mathrm{~A}}\right)$ under the control of the Myosin Light Chain (MLC) muscle specific promoter (Dobrowolny et al., 2008b) and 4 month old FVB (Friend leukemia virus B) (control strain). The animals were housed in a temperature-controlled $\left(22^{\circ} \mathrm{C}\right)$ room with a $12: 12 \mathrm{~h}$ light-dark cycle. All animal experiments were approved by the ethics committee of the Unit of Histology and Medical Embryology-Sapienza University of Rome- and were performed in accordance with the current version of the Italian Law on the Protection of Animals.

\section{RNA Isolation}

Total RNA was isolated from frozen lumbar spinal ventral tissue specimens using pestel homogenization, TRIzol reaction (Invitrogen, Carlsbad, CA, USA), and further on-column purification as previously described. The yield, quality and integrity of RNA were determined using the Agilent 2100 Bioanalyzer (Agilent Technologies, Palo Alto, CA, USA) as previously described (Bernardini et al., 2012).

\section{miRNA Analysis}

From each sample ( $n=4$ per experimental group), $600 \mathrm{ng}$ of total RNA was reverse transcribed using Taqman MicroRNA Reverse Transcription Kit with Megaplex Primer Pools A \& B (Applied Biosystems, Foster City, CA, USA). The cDNA was analyzed with Taqman Array Mouse MicroRNA A and B Cards Set version 3 (Applied Biosystems, Foster City, CA, USA) on the Applied Biosystems 7900HT qPCR system (Applied Biosystems, Foster City, CA, USA). Most of the MicroRNAs in the B Card were undetermined, therefore their analysis was omitted. The data was normalized by global mean normalization. SDS RQ Manager version 2.4 (Applied Biosystems, Foster City, CA, USA) was used to calculate $\mathrm{Ct}$ values (number of cycles required for the fluorescent signal to cross the set threshold), Ct values greater than 35 were considered as non-specific or undetected, and were filtered. MicroRNAs with missing values in at least 2 of 4 replicate samples per treatment group were removed from the dataset. 
The remaining Ct values were normalized using the $\mathrm{U} 6$ reference miRNA as suggested by manufacturer's protocol. The fold change between sample groups was calculated by subtracting the relative expression of the samples and then transforming the result to anti-log2 (i.e., $2^{\text {difference }}$ ).

Relevant information about the experiment, is available at http://www.ncbi.nlm.nih.gov/geo/ under accession GSE71193.

\section{miRNA Pathway Analysis}

The putative correlation between mRNAs and miRNAs was investigated through the MiRwalk 2.0, a comprehensive database that provides information on miRNA from Human, Mouse and Rat on their predicted as well as validated binding sites on their target genes. In particular we get information on miRNA produced by 8 established miRNA prediction programs on $3^{\prime}$ UTRs of all known genes of Mouse (Dweep et al., 2011; Bernardini et al., 2012).

miRNA predicted pathways were identified using DIANA (DNA Intelligence Analysis) tools (Vlachos et al., 2012).

DIANA-miRPath is a miRNA pathway analysis web-server that utilizes predicted miRNA targets (in CDS or $3^{\prime}$-UTR regions) provided by the DIANA-microT-CDS algorithm or even experimentally validated miRNA interactions derived from DIANA-TarBase v6.0. These interactions (predicted and/or validated) can be subsequently combined with sophisticated merging and meta-analysis algorithms. Essentially, this tool allows identifying all the predicted or experimentally validated miRNAs significantly targeting a selected pathway.

\section{Single qPCR Validation}

Single qPCR of miRNA 133a, 133b, 9, 29, 330, and 1 was performed to validate the performance of the Taqman arrays using Taqman probes from single tube Taqman microRNA assays with the diluted cDNA in a $10 \mu \mathrm{l}$ reaction volume, in triplicate for each assay, on the Applied Biosystems 7500HT system (Applied Biosystems, Foster City, CA, USA; $n=10$ per experimental group).

\section{Western Blot Analysis}

Protein extraction from both wild type and MLC/SOD1G93A transgenic spinal cord ( $n=5$ for each genotype) was performed in $10 \mathrm{mM}$ Tris, $150 \mathrm{mM}$ Sodium Chloride, $1 \%$ NP40, $0.1 \%$ SDS, $10 \%$ Glycerol, 1\% Deoxycholate, $1 \mathrm{mM}$ Phenylmethylsulfonyl Fluoride, $1 \mu \mathrm{g} / \mathrm{ml}$ Aprotinin, $1 \mu \mathrm{g} / \mathrm{ml}$ Leupeptin, $1 \mu \mathrm{g} / \mathrm{ml}$ Pepstatin, $1 \mathrm{mM}$ Sodium Orthovanadate, and $1 \mathrm{mM}$ Sodium Fluoride. Equal amounts of protein from each lysate were separated in SDS polyacrilamide gel and transferred onto a nitrocellulose membrane. Filters were blotted with antibodies against Pmp22 (Novus Biological Littleton, CO, USA. Cat. NBP1-67670), Mpz (Abcam, Cambridge, UK. Cat. Ab64685), and a-tubulin (Sigma, Saint Louis, MO, USA. Cat. T5168).

Pmp22 primary antibody was diluted 1:200 in TBST and used for blotting over night (o.n.) after 5\% milk saturation. Then, filter was incubated with secondary antibody (Goat anti-Rabbit IgG HRP-conjugated) (Bethyl, Montgomery, TX, USA. Cat. A120201P) in $1 \%$ milk for $1 \mathrm{~h}$.
Mpz primary antibody was diluted 1:500 in TBST and used for blotting o.n. after $10 \%$ milk saturation. Then, filter was incubated with secondary antibody (Goat anti-Mouse IgG HRPconjugated) (Bethyl, Montgomery, TX, USA. Cat. A90-516P) in $1 \%$ milk for $1 \mathrm{~h}$.

Tubulin was diluted 1:5000 in TBST and used for blotting o.n. after 5\% milk saturation. Then, filter was incubated with secondary antibody (Goat anti-Mouse IgG HRP-conjugated) (Bethyl Montgomery, TX, USA. Cat. A90-516P) in 1\% milk for $1 \mathrm{~h}$. Signals were acquired by ChemiDoc MP instrument (Bio-Rad, Hercules, CA, USA) and processed with Image Lab acquisition analysis software (Bio-Rad, Hercules, CA, USA).

Five independent samples for each group of animals have been used for densitometric analysis and protein level of $\alpha$-tubulin was used as control for equal protein loading.

\section{Microarray Analysis}

Total RNA was used to create the biotin-labeled library to be hybridized on GeneChip ${ }^{\circledR}$ Mouse Gene 2.0 ST Array (Affymetrix, Santa Clara, CA, USA) covering more than 26500 RefSeq coding transcripts and more than 3500 RefSeq noncoding transcripts, following the manufacturer protocol.

Briefly, double-stranded cDNA was synthesized routinely from less than 1 microgram of total RNA primed with a poly(dT) - T7 oligonucleotide. The cDNA was used in an in vitro transcription reaction in the presence of T7 RNA polymerase and biotin-labeled modified nucleotides during $16 \mathrm{~h}$ at $37^{\circ} \mathrm{C}$. Biotinylated cRNA was purified and then fragmented (35-200 nucleotides), together with hybridization controls and hybridized to the microarrays for $16 \mathrm{~h}$ at $45^{\circ} \mathrm{C}$. Using the GeneChip Fluidics Station 450 (Affymetrix, Santa Clara, CA, USA), the biotin-labeled cRNA was revealed by successive reactions with streptavidin R-phycoerythrin conjugate, biotinylated anti-streptavidin antibody and streptavidin R-phycoerythrin conjugate. The arrays were finally scanned in an Affymetrix GeneChip Scanner 7G Plus (Affymetrix, Santa Clara, CA, USA).

\section{Gene Expression Data Analysis-Differentially Expressed Gene List}

The CEL files that store the results of the intensity calculations on the pixel values collected from an Affymetrix scanner and result from the hybridization, were analyzed using Transcriptome Analysis Console (TAC) from Affymetrix (Affymetrix, Santa Clara, CA, USA). Gene-level calculation was performed by Robust Multichip Average to summarize probeset signal (Irizarry et al., 2003) and normalization by quantile sketch (Bolstad et al., 2003). A data table (rma), together with the relative CEL and relevant information about the experiment, is available at http:// www.ncbi.nlm.nih.gov/geo/ under accession GSE69582.

An unpaired One-way ANOVA was then used to identify differentially expressed genes, only the genes which met our criterion $(p<0.05$, fold change $>1.5)$ were selected in this study. The resulting gene list was then annotated according to the Gene Ontology (GO) database (www.geneontology.org). This allowed assigning a category to each gene in the list, according to 
three defined "ontologies" (i.e., terms representing gene product properties): cellular component, biological process and molecular function.

The expression of selected genes was quantified in real time PCR to obtain an independent validation of microarray data. Real time PCR was carried out as previously described elsewhere (Bernardini et al., 2012).

\section{Functional Categorization}

The gene expression list has been functionally categorized using the Database for Annotation, Visualization, and Integrated Discovery (DAVID; http://david.abcc.ncifcrf.gov/) (Sherman et al., 2007). The algorithms implemented in this software allow identifying over-represented gene ontology (GO) terms with respect to the total number of genes assayed and annotated. To this aim, DAVID applies a modified Fisher exact test, to establish if the proportion of genes falling into an annotation category significantly differs from the background group of genes. In addition, this tool enables the fine mapping of genes within welldefined signaling and/or metabolic pathways, classified in the Kyoto Encyclopedia of Genes and Genomes (KEGG) database (www.genome.jp/kegg/). The KEGG mapping tool was employed for the functional categorization of the gene regulatory networks. For this purpose, AffyGene IDs, corresponding to the genes in the selected list, were used as queries and the whole set of genes represented on the array was used as the background group. A $p<0.05$ was set.

The STRING (Search Tool for the Retrieval of Interacting Genes/Proteins) online software (Franceschini et al., 2013) was used to search interaction relationships of the proteins encoded by differential expressed genes.

\section{Histological Analysis}

Sciatic nerves were dissected and quickly fixed in 2.5\% glutaraldehyde in $0.1 \mathrm{M}$ sodium cacodylate for $2 \mathrm{~h}$, washed in $0.1 \mathrm{M}$ sodium cacodylate. Nerves were then post-fixed in $1 \%$ osmium in $0.1 \mathrm{M}$ sodium cacodylate for $1 \mathrm{~h}$, dehydrated in ethanol and embedded in epoxy resin. Samples were infiltrated with toluene and cut with a Leica ultracut $\mathrm{R}$ ultramicrotome (Leica Microsystems Inc. Buffalo Grove, IL) to obtain 2 um suitable for toluidine blue staining. Microscope using $40 \times$ lenses was employed, and images were processed using Axiovision Rel 4.8 (Zeiss, Oberkochen, D) representative images of sciatic nerve thin sections are shown cropped in Figure 5.

The g-ratio was calculated as $(\mathrm{dmax}+\mathrm{dmin}) / 2 /(\mathrm{Dmax}+$ Dmin) $/ 2$ and the analysis was performed for 200 fibers. Each diameter was calculated from the average of the major axis (Dmax: maximum fiber diameter; dmax:maximum axonal diameter) and the minor axis (Dmin: minimum fiber diameter; dmin: minimum axonal diameter), (Tsutsumi et al., 2014) and was measured using Image J software (Image J 1.36, National Institutes of Health, MD, USA).

\section{Statistical Analysis}

Statistical analysis, if not differently specified was performed using the Mann Whitney nonparametric test and significance was established for $p<0.05$.

\section{RESULTS}

\section{miRNA and mRNA Expression Profile of the Spinal Cord of the MLC/SOD1 ${ }^{\text {G93A }}$ Mice}

First, to identify deregulated miRNAs and transcripts in the spinal cord of MLC/SOD $1^{\mathrm{G} 93 \mathrm{~A}}$ transgenic mice we performed miRNA expression profiling and DNA microarray, using the Taqman array card and Affymetrix Mouse Gene 2.0 ST respectively (GSE71194). Among the analyzed miRNAs, 54 were significantly down-regulated $(p<0.05)$ in the spinal cord of $\mathrm{MLC} / \mathrm{SOD} 1^{\mathrm{G} 93 \mathrm{~A}}$ transgenic mice compared to wild type littermates (Table 1). Of note, the miRnome profiling revealed the down regulation of mir-330, mir-133, and mir-1, which are involved in denervation and reinnervation processes (Jeng et al., 2009; Tsutsumi et al., 2014). In addition mir-29 and mir-9 family member were downregulated and their deregulation was associated with different neurodegenerative diseases, including Huntington, Alzheimer, and Parkinson diseases (Saito and Saito, 2012; Tsutsumi et al., 2014) and demyelination-related diseases (Li and Yao, 2012; Tsutsumi et al., 2014). Real time PCR analysis was performed to validate the expression of these relevant miRNAs in the spinal cord of both wild type and MLC/SOD 1 G93A transgenic mice (Figure 1).

The microarray data, related to the expression of mRNA in the spinal cord of both wild type and MLC/SOD1 ${ }^{\text {G93A }}$ transgenic mice, were deposited in the GEO database (GSE69582) and the list of the selected significantly up-regulated and downregulated genes $(p<0.05 ; F C>1.5)$ is shown in Tables 2, 3 respectively. Among these genes, nine up-regulated transcripts are involved in the myelination process such as Peripheral myelin protein 22 (Pmp22), Myelin protein zero (Mpz), Periaxin (Prx), the Early growth response 2 (Egr2) genes, Desert hedgehog (Dhh), or encode for extracellular matrix molecules such as Matrilin 2 (Matn2), Gliomedin (Gldn), Claudin 19 (Cldn19), and Smoothelin (Smtn). Other relevant modulated genes include glycoproteins, such as Osteoglycin (Ogn) and dystroglicans, such as Dystrophin related protein 2 (Drp2), associated with the myelination process. The Mpz, Pmp22, Egr2, Prx mRNAs were selected for validation by qRT-PCR analysis and results are shown in Figure 2. Opalin, another gene associated to myelin structures, was down-regulated in the spinal cord of MLC/SOD1 ${ }^{\mathrm{G} 93 \mathrm{~A}}$ transgenic mice, compared to wild type littermates (Table 3). Opalin is expressed specifically in late stage of oligodendrocyte differentiation and has been shown to be dramatically reduced in a hypomyelination mouse model (Jiang et al., 2013; Tsutsumi et al., 2014).

In order to show changes in protein expression, as a result of altered microRNA/mRNA expression, we performed western blot analysis for Pmp22 and Mpz expression. We revealed a significant up-regulation of $\mathrm{Pmp} 22$ and $\mathrm{Mpz}$ proteins in the spinal cord of MLC/SOD $1^{\text {G93A }}$ compared to wild type littermates (Figure 3), further supporting the evidence that these factors are targets of specific microRNAs that control the denervation and reinnervation processes.

Taken together these results suggest that the muscle specific expression of the mutated isoform of the human SOD1 gene alters the expression of relevant markers of myelination in 
TABLE 1 | List of significantly down-regulated miRNAs in MLC/SOD1G93A spinal cord.

miRNA

Fold change

mmu-let-7b

mmu-let-7c

mmu-let-7d

mmu-let-7i

mmu-miR-100

mmu-miR-1

mmu-miR-106a

mmu-miR-106b

mmu-miR-10a

mmu-miR-10b

mmu-miR-128a

mmu-miR-129-3p

mmu-miR-129-5p

mmu-miR-133a

mmu-miR-133b

mmu-miR-15a

mmu-miR-15b

mmu-miR-17

mmu-miR-181a

mmu-miR-181c

mmu-miR-188-5p

mmu-miR-192

mmu-miR-194

mmu-miR-196b

mmu-miR-199a-3p

mmu-miR-19b

mmu-miR-20b

mmu-miR-23b

mmu-miR-26b

mmu-miR-29a

mmu-miR-29b

mmu-miR-29c

mmu-miR-301a

mmu-miR-30a

mmu-miR-30e

mmu-miR-324-5p

mmu-miR-328

mmu-miR-329

mmu-miR-330

mmu-miR-340-3p

mmu-miR-340-5p

mmu-miR-369-3p

mmu-miR-369-5p

mmu-miR-491

mmu-miR-497

mmu-miR-501-3p

mmu-miR-532-5p

mmu-miR-544

mmu-miR-652
$-4.97$

$-4.80$

$-3.85$

$-3.92$

$-4.31$

$-3.69$

$-5.23$

$-4.57$

$-4.07$

$-3.75$

$-4.69$

$-3.55$

$-7.58$

$-2.75$

$-3.94$

$-8.16$

$-3.84$

$-4.43$

$-4.35$

$-7.37$

$-4.20$

$-3.38$

$-3.60$

$-3.24$

$-4.67$

$-5.56$

$-6.42$

$-4.66$

$-5.25$

$-4.21$

$-6.33$

$-4.38$

$-4.21$

$-3.89$

$-3.37$

$-4.63$

$-3.75$

$-4.79$

$-8.50$

$-4.96$

$-3.83$

$-17.18$

$-3.64$

$-4.04$

$-6.93$

$-3.23$

$-4.21$

$-3.89$

$-5.73$
$\boldsymbol{P}$-value

0.04170

0.01392

0.02516

0.04791

0.02747

0.01614

0.02034

0.03639

0.01796

0.03798

0.02756

0.03832

0.00264

0.03894

0.00340

0.00251

0.02226

0.04014

0.02206

0.01568

0.03755

0.04185

0.01944

0.04461

0.01722

0.03463

0.02596

0.04223

0.01406

0.01571

0.01723

0.01636

0.04486

0.03876

0.03622

0.04475

0.04278

0.01774

0.00381

0.02453

0.03845

0.04086

0.02940

0.02354

0.00462

0.00474

0.04129

0.01407

0.02815

Continued)
TABLE 1 | Continued

\begin{tabular}{lcc}
\hline miRNA & Fold change & $\boldsymbol{P}$-value \\
\hline mmu-miR-7a & -8.35 & 0.00062 \\
mmu-miR-9 & -3.88 & 0.01623 \\
mmu-miR-92a & -4.34 & 0.01318 \\
mmu-miR-93 & -6.98 & 0.01638 \\
mmu-miR-99b & -3.56 & 0.02227 \\
\hline
\end{tabular}

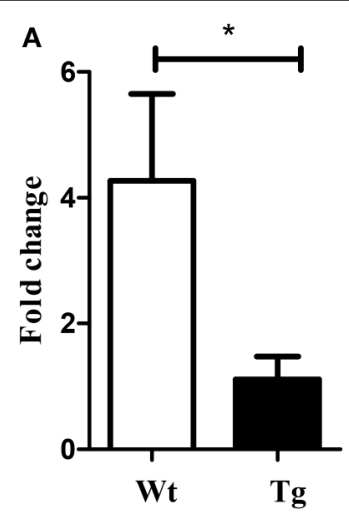

\section{B}

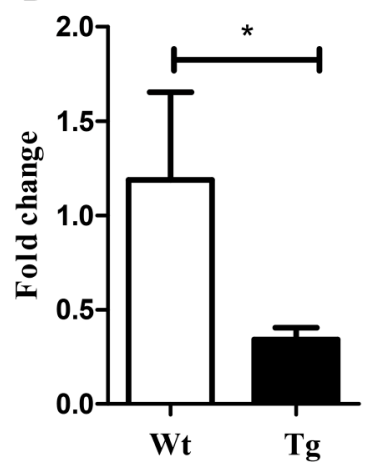

C
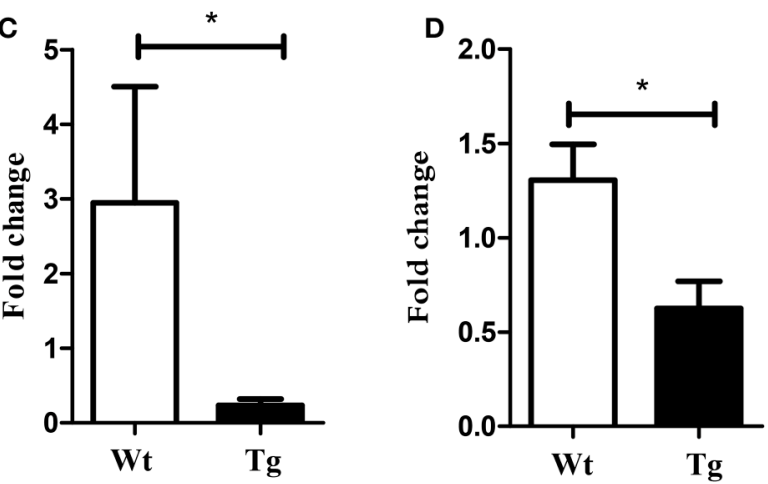

E

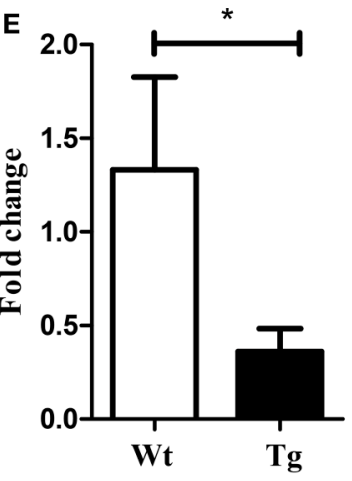

F

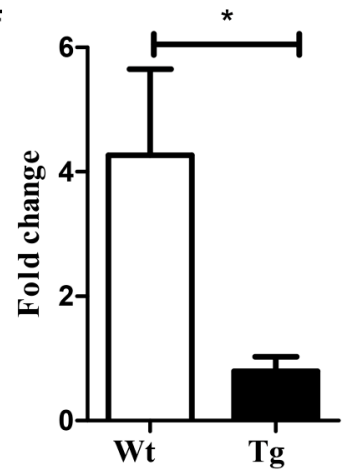

FIGURE 1 | Validation of selected miRNAs array data by Real-time PCR. Graphs indicate relative expression of (A) mir-133a (B) mir-133b (C) mir-9 (D) mir-29 (E) mir-330 (F) mir-1. White bar refers to wild type (Wt) and black bar to MLC/SOD1 G93A $(\mathrm{Tg})$. Values are expressed as mean $\pm \mathrm{SEM}$. ${ }^{*} p<0.05$ ( $n=10$ for each group). 
TABLE 2 | List of significantly up-regulated genes in MLC/SOD1G93A spinal cord.

\begin{tabular}{|c|c|c|c|c|c|c|c|}
\hline \multicolumn{4}{|c|}{ spinal cord. } & \multirow{3}{*}{$\begin{array}{l}\text { Gene } \\
\text { symbol }\end{array}$} & \multirow{3}{*}{ Gene name } & \multirow{3}{*}{ Fold change } & \multirow{3}{*}{$P$-value } \\
\hline & & & & & & & \\
\hline \multirow{2}{*}{$\begin{array}{l}\text { Gene } \\
\text { symbol }\end{array}$} & Gene name & Fold change & $P$-value & & & & \\
\hline & & & & \multirow[t]{2}{*}{ Sbspon } & \multirow{2}{*}{$\begin{array}{l}\text { somatomedin B and } \\
\text { thrombospondin }\end{array}$} & \multirow[t]{2}{*}{+1.66} & \multirow[t]{2}{*}{0.009017} \\
\hline Arhgap19 & Rho GTPase activating protein 19 & +2.14 & 0.018071 & & & & \\
\hline Arl4d & ADP-ribosylation factor-like 4D & +1.51 & 0.026430 & Slc36a2 & Solute carrier family 36, member 2 & +2.24 & 0.004210 \\
\hline Art3 & ADP-ribosyltransferase 3 & +1.63 & 0.003088 & \multirow[t]{2}{*}{ Smco3 } & \multirow{2}{*}{$\begin{array}{l}\text { Single-pass memb. protein } \\
\text { coiled-coil domains } 3\end{array}$} & \multirow[t]{2}{*}{+1.52} & \multirow[t]{2}{*}{0.004448} \\
\hline Ccdc37 & Coiled-coil domain containing 37 & +1.67 & 0.013866 & & & & \\
\hline Chrdl1 & Chordin-like 1 & +1.96 & 0.010071 & \multirow{4}{*}{$\begin{array}{l}\text { Smtn } \\
\text { Snhg3 } \\
\text { Snora17 }\end{array}$} & \multirow{4}{*}{$\begin{array}{l}\text { Smoothelin } \\
\text { Small nucleolar RNA host gene } 3 \\
\text { Small nucleolar RNA, H/ACA box } \\
17 \text {, }\end{array}$} & +1.55 & 0.028145 \\
\hline \multirow{2}{*}{ Cldn19 } & \multirow{2}{*}{ Claudin 19} & \multirow{2}{*}{+1.74} & \multirow{2}{*}{0.014558} & & & +2.65 & 0.016377 \\
\hline & & & & & & +2.74 & 0.027109 \\
\hline Ddn & Dendrin & +1.51 & 0.022150 & & & & \\
\hline Dhh & Desert hedgehog & +1.86 & 0.021201 & \multirow[t]{2}{*}{ Snora73b, } & \multirow{2}{*}{$\begin{array}{l}\text { Small nucleolar RNA, H/ACA box } \\
\text { 73b, }\end{array}$} & \multirow[t]{2}{*}{+2.23} & \multirow[t]{2}{*}{0.025905} \\
\hline Drp2 & Dystrophin related protein 2 & +1.79 & 0.012309 & & & & \\
\hline Egr2 & Early growth response 2 & +1.70 & 0.022549 & Sostdc1 & Sclerostin domain containing 1 & +1.68 & 0.007660 \\
\hline Gas2l3 & Growth arrest-specific 2 like 3 & +2.05 & 0.030748 & Tdpoz1 & TD and POZ domain containing 1 & +1.68 & 0.010976 \\
\hline Gimap1 & GTPase, IMAP family member 1 & +1.59 & 0.047143 & Tgfbi & Transforming growth factor, beta & +1.83 & 0.027616 \\
\hline Gldn & Gliomedin & +1.77 & 0.010186 & & induced & & \\
\hline Gulp1 & GULP, engulfment adaptor & +1.63 & 0.006082 & Th & Tyrosine hydroxylase & +1.72 & 0.025458 \\
\hline \multirow[t]{2}{*}{ Hif3a } & \multirow{2}{*}{$\begin{array}{l}\text { Hypoxia inducible factor } 3 \text {, alpha } \\
\text { subunit }\end{array}$} & +1.57 & 0.014288 & Vmn1r181 & Vomeronasal 1 receptor 181 & +1.51 & 0.042949 \\
\hline & & +1.51 & 0.014200 & $X \mid r 4 b$ & X-linked lymphocyte-regulated 4B & +1.88 & 0.005473 \\
\hline Hist2h2ab & Histone cluster 2, H2ab & +1.56 & 0.002544 & Zbtb16 & Zinc finger and BTB domain & +1.53 & 0.018846 \\
\hline Hoxc13 & Homeobox C13 & +1.60 & 0.045743 & & containıng 16 & & \\
\hline Hoxd10 & Homeobox D10 & +2.13 & 0.043521 & 7 & Z & +1.54 & 0.000034 \\
\hline
\end{tabular}

$\| 16$

Inmt

Krtap4-2

Matn2

Mertk

Mme

$\mathrm{Mpz}$

$\mathrm{Nr} 4 \mathrm{a} 2$

Ogn

Olfr102,

Olfr100

Olfr128

Olfr1283

Interleukin 16

Indolethylamine

$\mathrm{N}$-methyltransferase

$+1.64$

$+1.91$

$+1.54$

$+1.74$

$+1.80$

c-mer proto-oncogene tyrosine

kinase

Membrane metallo

endopeptidase

Myelin protein zero

$+1.64$

$+1.52$

group A, member 2

Osteoglycin

$+2.02$

$+2.07$

Olfactory receptor 128

$+1.78$

$+1.52$

$+2.01$

Pleckstrin homology domain

containing, family $\mathrm{A}$

Pmp22

Prrg4

Prx

Rabggtb,

Peripheral myelin protein 22

$+1.57$

$+1.66$

(G-carboxyglutamic acid) 4

$+1.98$

$+1.77$

RAB geranylgeranyl transferase, b

subunit

Rdh13

Retinol dehydrogenase 13

$+2.30$

(all-trans and 9-cis)

Rhox2h

Rpl31-ps4

Reproductive homeobox $2 \mathrm{H}$

$+1.72$

$+1.61$

$+1.60$
0.011144

0.016648

0.005207

0.006453

0.003151

0.018812

0.049548

0.013349

0.041760

0.001166

0.025072

0.030795

0.020446

0.022466

0.000427

0.019790

0.041637

0.034523

0.044442

0.004327

0.000512 the ventral root of the lumbar spinal cord of transgenic mice.

\section{Functional Annotation of the Identified Genes}

Functional annotation of differentially expressed genes was performed using the DAVID functional annotation tool, an integrated biological knowledge base and analytic tools aimed at systematically extracting biological meaning from large gene and protein lists (Huang da et al., 2009). Similar annotation contents were clustered into annotation group with biological meanings. The annotation results revealed that $2.6 \%$ of the gene list examined was consistent with OMIM disease categories, in particular 5 of 17 genes extracted were related to CharcotMarie-Tooth disease. On the other hand, the gene ontology analysis revealed that $39.3 \%$ of all gene list are significantly involved in biological processes category, $36.7 \%$ are part of cell components, and $39.8 \%$ are associated with molecular function categories. The nine interesting genes mentioned above were spread out in all the categories examined. The functional annotation clustering was done using the default parameters, Mus musculus as background and classification stringency was set as medium. Table $\mathbf{4}$ shows that the significantly modulated genes were involved in 5 functional clusters containing functional annotation for extracellular region, neuron development and differentiation, axonogenesis, and neurological system process. A further functional analysis was performed using STRING, a web base tool to explore potential protein-protein interaction,
TABLE 2 | Continued 
TABLE 3 | List of significantly down-regulated genes in MLC/SOD1 G93A spinal cord.

\begin{tabular}{|c|c|c|c|}
\hline Gene symbol & Gene name & $\begin{array}{l}\text { Fold } \\
\text { change }\end{array}$ & $P$-value \\
\hline Akr1c20 & $\begin{array}{l}\text { Aldo-keto reductase family } 1 \text {, } \\
\text { member } \mathrm{C}\end{array}$ & -1.64 & 0.031454 \\
\hline Aplnr & Apelin receptor & -1.78 & 0.020211 \\
\hline $\begin{array}{l}\text { C3, } \\
\text { LOC100048759 }\end{array}$ & $\begin{array}{l}\text { Complement component 3, } \\
\text { complement C3-like }\end{array}$ & -1.60 & 0.044240 \\
\hline Cyp2j12 & $\begin{array}{l}\text { Cytochrome P450, family 2, } \\
\text { subfamily j, polypeptide } 12\end{array}$ & -1.58 & 0.018190 \\
\hline Enpp6 & $\begin{array}{l}\text { Ectonucleotide } \\
\text { pyrophosphatase/ } \\
\text { phosphodiesterase } 6\end{array}$ & -1.58 & 0.015427 \\
\hline Frmpd4 & $\begin{array}{l}\text { FERM and PDZ domain } \\
\text { containing } 4\end{array}$ & -1.66 & 0.010645 \\
\hline Gbp4 & Guanylate binding protein 4 & -1.65 & 0.027557 \\
\hline Gm14257 & Predicted gene 14257 & -1.54 & 0.032704 \\
\hline $\begin{array}{l}\text { Gm15246, } \\
\text { BC022960 }\end{array}$ & $\begin{array}{l}\text { Predicted gene } 15246, \text { cDNA } \\
\text { sequence BC022960 }\end{array}$ & -1.84 & 0.029954 \\
\hline Gm3088 & Predicted gene 3088 & -1.61 & 0.048993 \\
\hline Klk6 & Kallikrein related-peptidase 6 & -1.70 & 0.005161 \\
\hline $\mathrm{mt}-\mathrm{Tt}$ & $\begin{array}{l}\text { Mitochondrially encoded tRNA } \\
\text { threonine }\end{array}$ & -1.52 & 0.036295 \\
\hline Nkx2-9 & $\begin{array}{l}\text { NK2 transcription factor related, } \\
\text { locus } 9 \text { (Drosophila) }\end{array}$ & -1.51 & 0.007001 \\
\hline Olfr611 & Olfactory receptor 611 & -4.75 & 0.000275 \\
\hline Olfr727 & Olfactory receptor 727 & -1.51 & 0.043050 \\
\hline Opalin & $\begin{array}{l}\text { Oligodendrocytic myelin } \\
\text { paranodal and inner loop prot. }\end{array}$ & -1.75 & 0.000707 \\
\hline Pi15 & Peptidase inhibitor 15 & -1.52 & 0.020502 \\
\hline Prok2 & Prokineticin 2 & -1.54 & 0.040329 \\
\hline Prps2 & $\begin{array}{l}\text { Phosphoribosyl pyrophosphate } \\
\text { synthetase } 2\end{array}$ & -1.60 & 0.034506 \\
\hline Serpina1c & $\begin{array}{l}\text { Serine peptidase inhibitor, clade } \\
\text { A, member } 1 \mathrm{C}\end{array}$ & -1.66 & 0.024381 \\
\hline Vmn1r37 & Vomeronasal 1 receptor 37 & -1.62 & 0.023405 \\
\hline Vmn2r71 & Vomeronasal 2, receptor 71 & -1.70 & 0.033296 \\
\hline Vnn1 & Vanin 1 & -1.65 & 0.010154 \\
\hline
\end{tabular}

confirming the interactions among Pmp22, Mpz, Prx, and Egr2 proteins (Figure 4).

\section{Correlation of Expression Profile between miRNAs and mRNA}

miRNAs are key regulators of all important biological processes and the level at which they act on coding mRNA (transcriptional, translational, etc.) is still debated; however gene repression by microRNAs at the level of mRNA translation is the most frequently reported mechanism (Morozova et al., 2012). Therefore, we further performed a systematic analysis on the interaction between significantly modulated miRNAs and mRNAs considering only miRNAs present in our list with an inverse mRNAs expression correlation. The analysis was performed using MiRwalk tool a comprehensive database that provides information on miRNA produced by 8 established
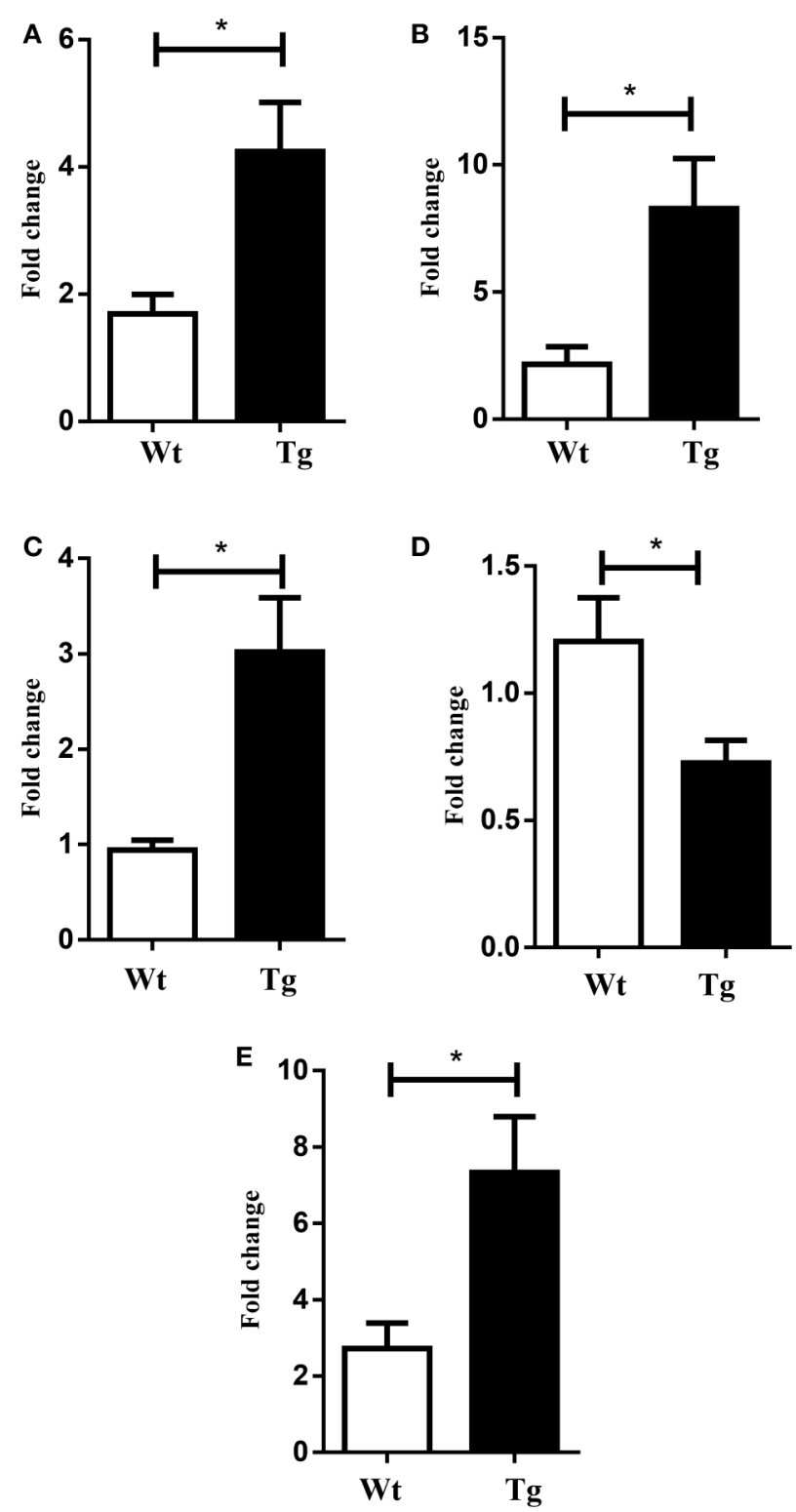

FIGURE 2 | Validation of selected mRNAs by Real-time PCR. Graphs (A) Pmp 22 (B) Prx (C) Mpz (D) Opalin (E) Egr2. White bar refers to wild type (Wt) and black bar to MLC/SOD1 G93A $(\mathrm{Tg})$. Values are expressed as mean $\pm \mathrm{SEM}$. ${ }^{*} p<0.05$ compared to Wt ( $n=4$ for each group).

miRNA prediction programs on $3^{\prime}$ UTRs of all known genes of mouse such as RNA22, miRanda, miRDB, TargetScan. Secondly, we ordered mRNAs on the basis of the number of putative targeting miRNAs and we observed that 36 genes were targeted from at least 1 to maximum 30 significantly modulated miRNAs; conversely 54 listed miRNAs targeted at least one to maximum of 11 significantly modulated genes; interestingly the most intriguing cross interaction involved genes and miRNAs of the myelination process (Table 5).

This finding clearly suggests that muscle specific overexpression of the SOD1 mutant gene induces the modulation of miRNAs and putative targeted-mRNAs involved in the 


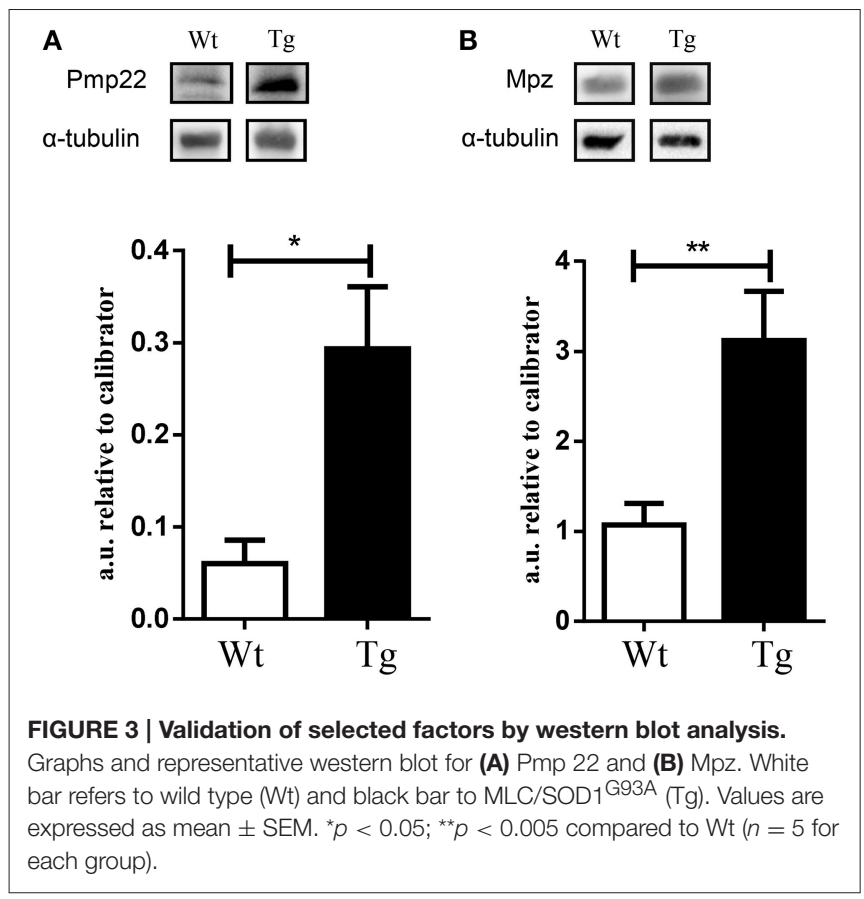

myelination process in the spinal cord of $\mathrm{MLC} / \mathrm{SOD} 1^{\mathrm{G} 93 \mathrm{~A}}$ transgenic mice.

\section{Mapping miRNAs to Signaling Pathways}

DIANA-mirPath is a web-based computational tool to identify KEGG signaling pathways regulated by miRNAs (see Materials and Methods section). The software compares each set of miRNA targets with all-known KEGG pathway and interestingly we observed high significance for axon guidance, glutamatergic synapse, and amyotrophic lateral sclerosis (ALS), meaning that these pathways are likely to be controlled by the altered miRNAs (Table 6).

\section{Muscle Specific Expression of SOD1 Mutant Gene Induces Hypomyelination in the Mice Sciatic Nerve}

Different reports indicate that ALS might be associated with motor nerve fiber (Echaniz-Laguna et al., 2006; Rajabally and Jacob, 2008; Ahdab et al., 2013). Previously, we demonstrated that the peripheral nerve was severely compromised in the global SOD1 ${ }^{\mathrm{G} 93 \mathrm{~A}}$ mutant mice, which displayed loss of Schmidt-Lantermann incisures, a disproportionately thick myelin sheath, abundant double onion bulb structures, and increased demyelinated axons (Dobrowolny et al., 2008a). To assess whether muscle specific expression of SOD1 mutant gene induces a peripheral alteration of axon myelination we calculated the ratio of axon diameter to total fiber diameter (g-ratio) (Michailov et al., 2004). The Figure 5A shows the cross-sections of wild type and MLC/SOD1 ${ }^{\mathrm{G} 93 \mathrm{~A}}$ sciatic nerves stained with toluidine blue. The histological analysis of the peripheral nerve of the MLC/SOD1 ${ }^{\mathrm{G} 93 \mathrm{~A}}$ displayed the presence of double onion bulb structures. Moreover, the g-ratio in the transgenic sciatic
TABLE 4 | Functional annotation clustering of up-regulated mRNAs.

\begin{tabular}{lccc}
\hline Annotation cluster $\mathbf{1}$ & \multicolumn{2}{c}{ Enrichment score: $\mathbf{1 . 8 5}$} \\
\cline { 1 - 1 } Category & Count & $\boldsymbol{P}$-value \\
\hline GOTERM_CC_FAT extracellular region & 15 & $2.7 \mathrm{E}-3$ \\
GOTERM_CC_FAT extracellular region part & 8 & $2.4 \mathrm{E}-2$ \\
GOTERM_CC_FAT extracellular space & 6 & $4.2 \mathrm{E}-2$ \\
\hline
\end{tabular}

Annotation cluster 3

Enrichment score: 0.95

\begin{tabular}{lll}
\hline GOTERM_BP_FAT neuron development & 5 & $1.4 \mathrm{E}-2$ \\
GOTERM_MF_FAT transcription factor activity & 7 & $3.8 \mathrm{E}-2$ \\
GOTERM_BP_FAT neuron differentiation & 5 & $4.0 \mathrm{E}-2$
\end{tabular}

Annotation cluster 4

Enrichment score: 0.87

\begin{tabular}{lll}
\hline GOTERM_CC_FAT extracellular region part & 8 & $2.4 \mathrm{E}-2$
\end{tabular}

Annotation cluster 5

Enrichment score: 0.8

GOTERM_BP_FAT locomotory behavior

7.3E-3

GOTERM_BP_FAT behavior

GOTERM_BP_FAT pattern specification process

GOTERM_BP_FAT neuron development

GOTERM_BP_FAT response to endogenous stimulus

GOTERM_BP_FAT regionalization

GOTERM_MF_FAT transcription factor activity

GOTERM_BP_FAT neuron differentiation

$\begin{array}{ll}5 & 7.3 \mathrm{E}-3 \\ 6 & 9.6 \mathrm{E}-3\end{array}$

$5 \quad 1.3 \mathrm{E}-2$

$5 \quad 1.4 \mathrm{E}-2$

$42.2 \mathrm{E}-2$

$4 \quad 3.2 \mathrm{E}-2$

$7 \quad 3.8 \mathrm{E}-2$

$5 \quad 4.0 \mathrm{E}-2$

Annotation cluster 7

Enrichment score: 0.53

GOTERM_BP_FAT neurological system process

11

$3.9 \mathrm{E}-2$

Annotation cluster means a group of terms having similar biological functions. Enrichment score is the rank of the annotation cluster biological significance. Count column refers to number of genes involved in the same category.

nerves was significantly increased (Figure 5B) independently of the axonal diameter (Figure 5C), indicating that muscle specific expression of SOD1 mutant gene induces hypomyelination in the sciatic nerve of transgenic mice.

\section{DISCUSSION}

The functional interplay between muscle and nerve is crucial for the survival and function of both tissues throughout life and ALS represents one of the best examples of impaired interplay between the two tissues (Musarò, 2013). Although, there have been significant advances in our understanding of the biology of ALS, no consensus has emerged as to which cells, tissues and pathways are directly affected by mutant SOD1. Motor neurons degeneration and muscle atrophy are the major pathological processes associated with ALS consistent with the role of nerve activity in muscle homeostasis and remodeling (Munson et al., 1997; Pette, 2001; Schiaffino et al., 2007). However, other cells may be involved in the pathogenesis of ALS and alteration in skeletal muscle homeostasis might represent one of the critical mediators of motor neuron degeneration. This hypothesis has been recently investigated by different groups, and we 


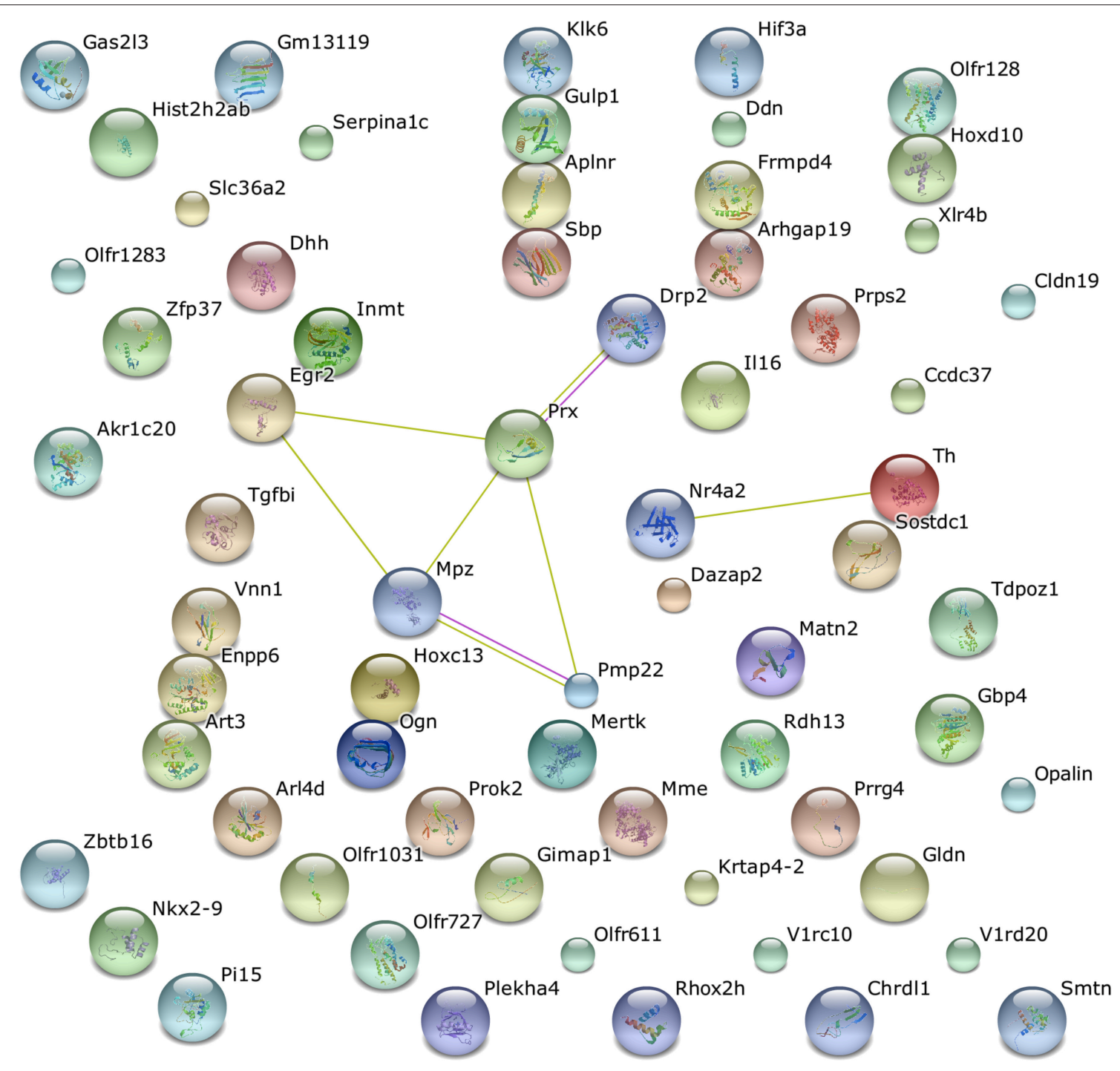

FIGURE 4 | STRING analysis of pathway enrichment and interaction in the altered mRNAs of the MLC/SOD1G93A transgenic mice. Protein products of five modulated mRNAs (Drp2, Prx, Egr2, Mpz, and Pmp22) were involved in eight interactions.

recently demonstrated that muscle selective expression of SOD1 mutation causes pathological alterations in skeletal muscle and induces pre-symptomatic sign of ALS at the level of spinal cord (Dobrowolny et al., 2008b).

In the present study, we extended our previous work and analyzed whether muscle-specific expression of $\mathrm{SOD}^{\mathrm{G}}{ }^{\mathrm{G}} \mathrm{A}$ is able to affect the expression of non-coding RNA and mRNA in the spinal cord of $\mathrm{MLC} / \mathrm{SOD} 1^{\mathrm{G} 93 \mathrm{~A}}$ transgenic mice, compared with wild type littermates. Our findings are in agreement with previous studies showing that skeletal muscle is also a source of signals that influence neuron survival, axonal growth and maintenance of synaptic connections (Funakoshi et al., 1995; Dobrowolny et al., 2005). In the absence of trophic support, muscle can have a negative impact on the nervous system, and therefore, can contribute to the alteration in the functional connection between muscle and nerve.
The major findings of our study indicate that muscle-specific expression of SOD1 ${ }^{\mathrm{G} 93 \mathrm{~A}}$ can modulate relevant molecules of the genetic and epigenetic circuitry of the myelin homeostasis in the spinal cord of transgenic mice. In particular, we found down regulation of mir-1, mir-330, mir-29, mir-133, and mir-9 family members, whose dysregulation can have profound effects on neuronal physiology and pathology, including Huntington, Alzheimer, and Parkinson diseases (Saito and Saito, 2012). Although, it is known that deregulation of specific miRNAdependent regulatory circuitries correlates with the initiation and progression of several neurological disorders, the underlying mechanisms of these phenomena are still not fully understood.

Here we revealed the alteration of specific microRNAs and potential mRNA targets, which might be part of the short circuit that disrupts the communication between skeletal muscle and nerve and that might be part of the so called dying back 
TABLE 5 | List of selected genes and corresponding regulated miRNAs.

\begin{tabular}{|c|c|c|c|}
\hline Gene & miRNA & miRNA & Gene \\
\hline \multirow[t]{3}{*}{ Egr2 } & mmu-miR-93 & mmu-miR-1 & Cldn19 \\
\hline & mmu-miR-17 & & DrP2 \\
\hline & mmu-miR-20b & & $\mathrm{Mpz}$ \\
\hline \multirow[t]{9}{*}{ Mpz } & mmu-miR-1 & & $\mathrm{Nr} 4 \mathrm{a} 2$ \\
\hline & mmu-miR-129-3p & & Ogn \\
\hline & mmu-miR-133a & & $\operatorname{Prx}$ \\
\hline & mmu-miR-133b & & Drp2 \\
\hline & mmu-miR-192 & mmu-miR-9 & Dhh \\
\hline & mmu-miR-30a & & Mme \\
\hline & mmu-miR-30e & & Pmp22 \\
\hline & mmu-miR-329 & & Prx \\
\hline & mmu-miR-491 & & Rdh13 \\
\hline \multirow[t]{9}{*}{ Pmp22 } & mmu-miR-26b & & Tgfbi \\
\hline & mmu-miR-29a & mmu-miR-15 & Cldn19 \\
\hline & mmu-miR-29b & & Gldn \\
\hline & mmu-miR-29c & & Prx \\
\hline & mmu-miR-330 & & Rdh13 \\
\hline & mmu-miR-340-3p & & \\
\hline & mmu-miR-501-3p & mmu-miR-29 & Drp2 \\
\hline & mmu-miR-544 & & Gldn \\
\hline & mmu-miR-9 & & Pmp22 \\
\hline \multirow[t]{19}{*}{$\operatorname{Prx}$} & mmu-miR-1 & mmu-miR-133 a-b & Cldn19 \\
\hline & mmu-miR-106a & & $\mathrm{Mpz}$ \\
\hline & mmu-miR-10a & & Rdh13 \\
\hline & mmu-miR-10b & & \\
\hline & mmu-miR-128 & & \\
\hline & mmu-miR-129-3p & & \\
\hline & mmu-miR-15a & & \\
\hline & mmu-miR-15b & & \\
\hline & mmu-miR-17 & & \\
\hline & mmu-miR-188-5p & & \\
\hline & mmu-miR-206 & & \\
\hline & mmu-miR-20b & & \\
\hline & mmu-miR-328 & & \\
\hline & mmu-miR-330 & & \\
\hline & mmu-miR-491 & & \\
\hline & mmu-miR-497 & & \\
\hline & mmu-miR-7a & & \\
\hline & mmu-miR-9 & & \\
\hline & mmu-miR-93 & & \\
\hline
\end{tabular}

Schematic representation of the inversely correlated miRNAs and predicted targets based on Mirwalk analysis.

phenomenon (Dadon-Nachum et al., 2011). In particular, among transcripts that are potential targets of the altered miRNAs, we revealed the presence of relevant markers of myelin homeostasis that are modulated by muscle restricted expression of SOD1 mutation inducing hypomyelination of the peripheral nerve. The up-regulation of Pmp22 and Mpz proteins in the spinal cord of MLC/SOD $1{ }^{\mathrm{G} 93 \mathrm{~A}}$ paralleled that of mRNA expression and supports the evidence that these factors are molecular targets of microRNAs, such as miR-1, miR-9, miR-133, and miR-330, that resulted differently modulated in the spinal cord of $\mathrm{MLC} / \mathrm{SOD} 1^{\mathrm{G} 93 \mathrm{~A}}$ mice compared to wild type littermates.
TABLE 6 | Signaling pathways predicted to be regulated by miRNAs.

\begin{tabular}{lll}
\hline KEGG Pathway & P-value & Number of miRNA \\
\hline PI3K-Akt signaling pathway & 5.368871 e-57 & 48 \\
MAPK signaling pathway & $2.427724 \mathrm{e}-54$ & 46 \\
Focal adhesion & $9.558692 \mathrm{e}-36$ & 44 \\
Wnt signaling pathway & $1.247526 \mathrm{e}-33$ & 42 \\
Axon guidance & $3.168282 \mathrm{e}-33$ & 42 \\
Ubiquitin mediated proteolysis & $3.031889 \mathrm{e}-27$ & 37 \\
Neurotrophin signaling pathway & $3.031889 \mathrm{e}-27$ & 42 \\
Insulin signaling pathway & $1.208302 \mathrm{e}-26$ & 45 \\
ErbB signaling pathway, & $6.307645 \mathrm{e}-22$ & 46 \\
Transcriptional misregulation in cancer & $9.134568 \mathrm{e}-22$ & 45 \\
Protein processing in endoplasmic reticulum & $1.448874 \mathrm{e}-21$ & 40 \\
TGF-beta signaling pathway & $4.891741 \mathrm{e}-21$ & 36 \\
HIF-1 signaling pathway & $2.055494 \mathrm{e}-20$ & 43 \\
Adherens junction & $4.208319 \mathrm{e}-16$ & 36 \\
Jak-STAT signaling pathway & $2.747301 \mathrm{e}-15$ & 39 \\
mTOR signaling pathway & $1.21693 \mathrm{e}-14$ & 38 \\
VEGF signaling pathway & $1.531543 \mathrm{e}-13$ & 38 \\
Glutamatergic synapse & $1.114872 \mathrm{e}-10$ & 38 \\
Amyotrophic lateral sclerosis (ALS) & $7.053373 \mathrm{e}-10$ & 34 \\
Notch signaling pathway & $9.441105 \mathrm{e}-09$ & 29 \\
Toll-like receptor signaling pathway & $3.1133 \mathrm{e}-08$ & 35 \\
Circadian rhythm & $8.717738 \mathrm{e}-08$ & 32 \\
Dorso-ventral axis formation & $3.707892 \mathrm{e}-06$ & 28 \\
Adipocytokine signaling pathway & $8.449379 \mathrm{e}-06$ & 33 \\
Dopaminergic synapse & $1.421481 \mathrm{e}-05$ & 38 \\
Dilated cardiomyopathy & $6.37123 \mathrm{e}-05$ & 35 \\
Cholinergic synapse & 0.0001426234 & 36 \\
Protein digestion and absorption & 0.0004322993 & 35 \\
mRNA surveillance pathway & 0.0005316409 & 36 \\
Cytokine-cytokine receptor interaction & 0.00200525 & 43 \\
Calcium signaling pathway & 0.03407104 & 42 \\
D-Glutamine and D-glutamate metabolism & 0.03934887 & 5 \\
Carbohydrate digestion and absorption & 0.0460262 & 20 \\
\hline
\end{tabular}

It has been demonstrated that Myelin protein zero is upregulated in oligodendroglia following axonal injury (da Silva et al., 2015) and Periaxin is expressed by myelinating Schwann cells in association with the dystroglycan complex through Drp2 (Sherman et al., 2012). Moreover, Pmp22 exhibits a dose dependent function as both overexpression and deletion result in hereditary neuropathy (Han et al., 2013). Dhh is essential for the structural and functional integrity of the peripheral nerve (Sharghi-Namini et al., 2006) and Osteglycine, Claudin -19 and Smoothelin together with Pmp22 and Prx are associated with reduced myelination of the spinal cord in the hypogravity motor syndrome (Chelyshev et al., 2014). Moreover, mutations in Mpz, Pmp22, Egr2, and Prx are associated with Charcot-Marie-Tooth neuropathy in which the over expression of these genes leads to defective myelination processes.

Recently, some specific miRNAs, such as miR-9, miR-23, and miR-29a, were found to participate in the regulation of oligodendrocyte differentiation and myelin maintenance, as well as in the pathogenesis of demyelination-related diseases. These 


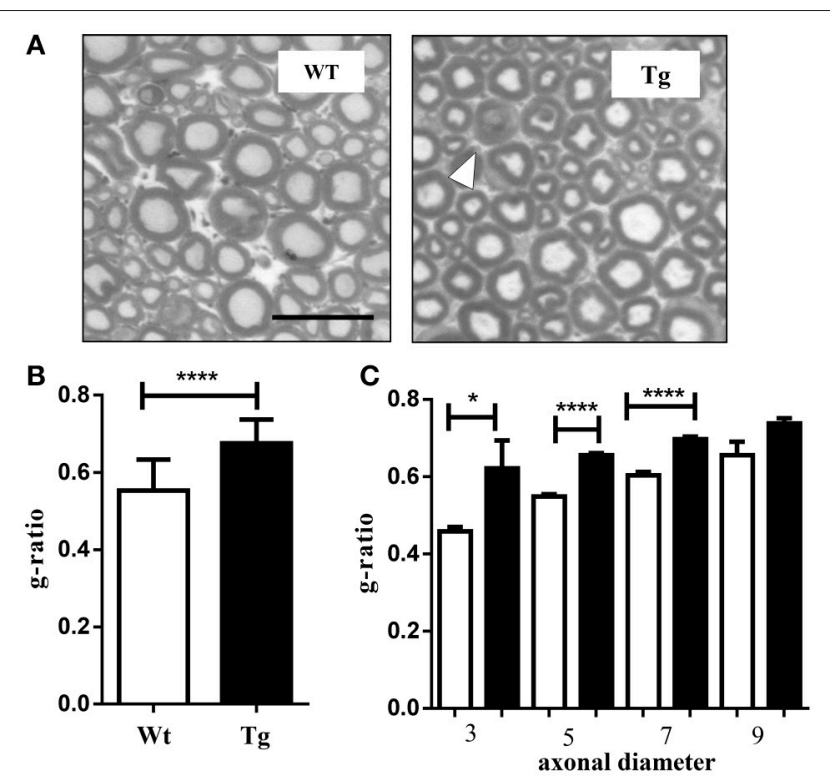

FIGURE 5 | Hypomyelination in the sciatic nerve of MLC/SOD1G93A mice. (A) Photographs showing the mouse sciatic nerve strained with toluidine blue. White arrowhead indicates onion bulb structure (40X magnification) Scale bar $=20 \mu \mathrm{m}$. (B) The average g-ratios. (C) The average g-ratio for each axonal diameter (1-3, 3-5, 5-7, >7 $\mu \mathrm{m})$. Values are expressed as mean \pm SEM fibers. ${ }^{*} p<0.05 ;{ }^{* * *} p<0.0001$ compared to wild type (Wt).

miRNAs control their target mRNAs and are involved in the pathogenesis of demyelination-related diseases (Lau et al., 2008; Verrier et al., 2009; Li and Yao, 2012).

Regarding the mRNA-miRNA interplay, most of the putative interactions proposed here have not been previously described and deserve further investigation. Interestingly, the functional interaction of miR-9 with Peripheral myelin protein 22 (Pmp22) mRNA has been already demonstrated (Lau et al., 2008). In particular, miR-9 is down-regulated during oligodendrocyte differentiation and its expression level inversely correlates with the expression of its targets Pmp22. Moreover, it has been described that the inhibition of endogenous miR-29 overrides the miRNA-mediated repression of Pmp22 cultured Schwann cells during both development and post-crush injury (Verrier et al., 2009). Therefore, these data suggest that myelin gene expression is regulated by miRNAs.

Here we observed that the muscle specific expression of the SOD1 mutant gene induces the deregulation of both mir-9

\section{REFERENCES}

Ahdab, R., Créange, A., Saint-Val, C., Farhat, W. H., and Lefaucheur, J. P. (2013). Rapidly progressive amyotrophic lateral sclerosis initially masquerading as a demyelinating neuropathy. Neurophysiol. Clin. 43, 181-187. doi: 10.1016/j.neucli.2013.05.001

Bernardini, C., Barba, M., Tamburrini, G., Massimi, L., Di Rocco, C., Michetti, F., et al. (2012). Gene expression profiling in human craniosynostoses: a tool to investigate the molecular basis of suture ossification. Childs Nerv. Syst. 28, 1295-1300. doi: 10.1007/s00381-0121780-2 and 29 together with myelin alteration in the sciatic nerve. These data have been validated by histological analysis of the sciatic nerve that shows a reduction in the myelin sheath in the MLC/SOD1 ${ }^{\mathrm{G} 93 \mathrm{~A}}$ mice model. In this regard it is interesting to note that the classical oligodendrocyte markers were downregulated (considering a fold change $<1.5$ olig2 $F C=-1.3$ $p=0.02$; Nkx2-2 FC $=-1.36 ; p=0.008$; Mag $\mathrm{FC}=-1.39$, $p=0.01)$. Therefore the reduction in oligodendrogenesis could be correlated with the hypomyelination in the MLC/SOD ${ }^{\mathrm{G} 93 \mathrm{~A}}$ mouse model.

In the spinal cord of $\mathrm{SOD} 1^{\mathrm{G} 93 \mathrm{~A}}$ transgenic mice that ubiquitously express the mutant SOD1 gene, the miRNA-9 expression is up-regulated (Zhou et al., 2013). In this model there is extensive degeneration of gray matter oligodendrocytes in the spinal cord prior to disease onset; actually new oligodendrocytes were formed but they failed to mature, resulting in progressive demyelination (Kang et al., 2013). The difference in the expression levels of miRNA-9 between the global SOD1 ${ }^{\mathrm{G} 93 \mathrm{~A}}$ and MLC/SOD1 ${ }^{\mathrm{G} 93 \mathrm{~A}}$ mice is likely due to $\mathrm{SOD} 1^{\mathrm{G} 93 \mathrm{~A}}$ gene ubiquitously expressed in all tissues, including muscle, motor neurons and glia. This might generate a synergistic toxic effect, leading to a more severe phenotype.

Although, most of the miRNA and mRNA inverted correlations proposed here have not been yet validated, the elevated number of potential interactions strongly points toward a role of skeletal muscle in myelin homeostasis. Nevertheless, although these results point toward some mechanisms of action of muscle specific expression of SOD $1^{\mathrm{G} 93 \mathrm{~A}}$ at the level of spinal cord, the elevated number of potential targets of the analyzed miRNAs make these mechanisms only mere suggestions. Additional studies will define which cell types, at the levels of spinal cord of MLC/SOD1 ${ }^{\text {G93A }}$ mice, specifically exhibit changes in microRNAs and mRNAs.

Overall our study provides additional insights into the effects of muscle selective expression of SOD1 ${ }^{\mathrm{G} 93 \mathrm{~A}}$ on nerve homeostasis and reveals the potential miRNA and mRNA signature associated with the dying back phenomenon.

\section{ACKNOWLEDGMENTS}

We thank C. Nicoletti for technical support, B. LozanoskaOchser for manuscript English editing. This work was supported by ASI (grant no. 2013-088-R.0), Fondazione Roma, Telethon (GGP14066), Nando Peretti Foundation, and PRIN (grant no. 2010R8JK2X). The authors do not have financial interest in relation to this submission.

Boillée, S., Yamanaka, K., Lobsiger, C. S., Copeland, N. G., Jenkins, N. A., Kassiotis, G., et al. (2006). Onset and progression in inherited ALS determined by motor neurons and microglia. Science 312, 1389-1392. doi: 10.1126/science.1123511

Bolstad, B. M., Irizarry, R. A., Astrand, M., and Speed, T. P. (2003). A comparison of normalization methods for high density oligonucleotide array data based on variance and bias. Bioinformatics 19, 185-193. doi: 10.1093/bioinformatics/19.2.185

Chelyshev, Y. A., Muhamedshina, Y. O., Povysheva, T. V., Shaymardanova, G. F., Rizvanov, A. A., Nigmetzyanova, M. V., et al. (2014). Characterization of spinal cord glial cells in a model of hindlimb unloading in mice. Neuroscience 280, 328-339. doi: 10.1016/j.neuroscience.2014.09.004 
Dadon-Nachum, M., Melamed, E., and Offen, D. (2011). The "dying-back" phenomenon of motor neurons in ALS. J. Mol. Neurosci. 43, 470-477. doi: 10.1007/s12031-010-9467-1

da Silva, J. T., Santos, F. M., Giardini, A. C., Martins, D. O., de Oliveira, M. E., Ciena, A. P., et al. (2015). Neural mobilization promotes nerve regeneration by nerve growth factor and myelin protein zero increased after sciatic nerve injury. Growth Factors 33, 8-13. doi: 10.3109/08977194.2014.953630

Dobrowolny, G., Aucello, M., Molinaro, M., and Musarò, A. (2008a). Local expression of mIgf-1 modulates ubiquitin, caspase and CDK5 expression in skeletal muscle of an ALS mouse model. Neurol. Res. 30, 131-136. doi: $10.1179 / 174313208 X 281235$

Dobrowolny, G., Aucello, M., Rizzuto, E., Beccafico, S., Mammucari, C., Boncompagni, S., et al. (2008b). Skeletal muscle is a primary target of SOD ${ }^{\mathrm{G} 93 \mathrm{~A}}$-mediated toxicity. Cell Metab. 8, 425-436. doi: 10.1016/j.cmet.2008.09.002

Dobrowolny, G., Giacinti, C., Pelosi, L., Nicoletti, C., Winn, N., Barberi, L., et al. (2005). Muscle expression of a local Igf-1 isoform protects motor neurons in an ALS mouse model. J. Cell Biol. 168, 193-199. doi: 10.1083/jcb.200407021

Dupuis, L., Gonzalez de Aguilar, J. L., Echaniz-Laguna, A., Eschbach, J., Rene, F., Oudart, H., et al. (2009). Muscle mitochondrial uncoupling dismantles neuromuscular junction and triggers distal degeneration of motor neurons. PLoS ONE 4:e5390. doi: 10.1371/journal.pone.0005390

Dweep, H., Sticht, C., Pandey, P., and Gretz, N. (2011). miRWalk-database: prediction of possible miRNA binding sites by "walking" the genes of three genomes. J. Biomed. Inform. 44, 839-847. doi: 10.1016/j.jbi.2011.05.002

Echaniz-Laguna, A., Degos, B., Mohr, M., Kessler, R., Urban-Kraemer, E., and Tranchant, C. (2006). A study of three patients with amyotrophic lateral sclerosis and a polyneuropathy resembling CIDP. Muscle Nerve 33, 356-362. doi: 10.1002/mus.20475

Franceschini, A., Szklarczyk, D., Frankild, S., Kuhn, M., Simonovic, M., Roth, A., et al. (2013). STRING v9.1: protein-protein interaction networks, with increased coverage and integration. Nucleic Acids Res. 41, D808-D815. doi: 10.1093/nar/gks1094

Funakoshi, H., Belluardo, N., Arenas, E., Yamamoto, Y., Casabona, A., Persson, H., et al. (1995). Muscle-derived neurotrophin-4 as an activity-dependent trophic signal for adult motor neurons. Science 268, 1495-1499. doi: 10.1126/science.7770776

Han, H., Myllykoski, M., Ruskamo, S., Wang, C., and Kursula, P. (2013). Myelin-specific proteins: a structurally diverse group of membrane-interacting molecules. Biofactors 39, 233-241. doi: 10.1002/biof.1076

Huang da, W., Sherman, B. T., and Lempicki, R. A. (2009). Systematic and integrative analysis of large gene lists using DAVID bioinformatics resources. Nat. Protoc. 4, 44-57. doi: 10.1038/nprot.2008.211

Irizarry, R. A., Bolstad, B. M., Collin, F., Cope, L. M., Hobbs, B., and Speed, T. P. (2003). Summaries of Affymetrix GeneChip probe level data. Nucleic Acids Res. 31:e15. doi: 10.1093/nar/gng015

Jeng, S. F., Rau, C. S., Liliang, P. C., Wu, C. J., Lu, T. H., Chen, Y. C., et al. (2009). Profiling muscle-specific microRNA expression after peripheral denervation and reinnervation in a rat model. J. Neurotrauma 26, 2345-2353. doi: 10.1089/neu.2009.0960

Jiang, W., Yang, W., Yang, W., Zhang, J., Pang, D., Gan, L., et al. (2013). Identification of Tmem10 as a novel late-stage oligodendrocytes marker for detecting hypomyelination. Int. J. Biol. Sci. 10, 33-42. doi: 10.7150/ijbs.7526

Julien, J. P. (2007). ALS: astrocytes move in as deadly neighbors. Nat. Neurosci. 10, 535-537. doi: 10.1038/nn0507-535

Kablar, B., and Rudnicki, M. A. (1999). Development in the absence of skeletal muscle results in the sequential ablation of motor neurons from the spinal cord to the brain. Dev. Biol. 208, 93-109. doi: 10.1006/dbio.1998.9184

Kang, S. H., Li, Y., Fukaya, M., Lorenzini, I., Cleveland, D. W., Ostrow, L. W., et al. (2013). Degeneration and impaired regeneration of gray matter oligodendrocytes in amyotrophic lateral sclerosis. Nat. Neurosci. 16, 571-579. doi: 10.1038/nn.3357

Lau, P., Verrier, J. D., Nielsen, J. A., Johnson, K. R., Notterpek, L., and Hudson, L. D. (2008). Identification of dynamically regulated microRNA and mRNA networks in developing oligodendrocytes. J. Neurosci. 28, 11720-11730. doi: 10.1523/JNEUROSCI.1932-08

Li, J. S., and Yao, Z. X. (2012). MicroRNAs: novel regulators of oligodendrocyte differentiation and potential therapeutic targets in demyelination-related diseases. Mol. Neurobiol. 45, 200-212. doi: 10.1007/s12035-011-8231-z
Michailov, G. V., Sereda, M. W., Brinkmann, B. G., Fischer, T. M., Haug, B., Birchmeier, C., et al. (2004). Axonal neuregulin-1 regulates myelin sheath thickness. Science 304, 700-703. doi: 10.1126/science. 1095862

Morozova, N., Zinovyev, A., Nonne, N., Pritchard, L. L., Gorban, A. N., and HarelBellan, A. (2012). Kinetic signatures of microRNA modes of action. RNA 18, 1635-1655. doi: 10.1261/rna.032284.112

Munson, J. B., Foehring, R. C., Mendell, L. M., and Gordon, T. (1997). Fastto-slow conversion following chronic low-frequency activation of medial gastrocnemius muscle in cats. II. Motoneuron properties. J. Neurophysiol. 77, 2605-2615.

Musarò, A. (2010). State of the art and the dark side of amyotrophic lateral sclerosis. World J. Biol. Chem. 1, 62-68. doi: 10.4331/wjbc.v1.i5.62

Musarò, A. (2013). Understanding ALS: new therapeutic approaches. FEBS J. 280, 4315-4322. doi: 10.1111/febs.12087

Pette, D. (2001). Historical perspectives: plasticity of mammalian skeletal muscle. J. Appl. Physiol. (1985) 90, 1119-1124.

Rajabally, Y. A., and Jacob, S. (2008). Chronic inflammatory demyelinating polyneuropathy-like disorder associated with amyotrophic lateral sclerosis. Muscle Nerve 38, 855-860. doi: 10.1002/mus.21010

Rosen, D. R. (1993). Mutations in $\mathrm{Cu} / \mathrm{Zn}$ superoxide dismutase gene are associated with familial amyotrophic lateral sclerosis. Nature 364, 362. doi: $10.1038 / 362059 \mathrm{a} 0$

Saito, Y., and Saito, H. (2012). MicroRNAs in cancers and neurodegenerative disorders. Front. Genet. 3:194. doi: 10.3389/fgene.2012.00194

Schiaffino, S., Sandri, M., and Murgia, M. (2007). Activity-dependent signaling pathways controlling muscle diversity and plasticity. Physiol. (Bethesda.) 22, 269-278. doi: 10.1152/physiol.00009

Sharghi-Namini, S., Turmaine, M., Meier, C., Sahni, V., Umehara, F., Jessen, K. R., et al. (2006). The structural and functional integrity of peripheral nerves depends on the glial-derived signal desert hedgehog. J. Neurosci. 26, 6364-6376. doi: 10.1186/1471-2105-8-426

Sherman, B. T., Huang da, W., Tan, Q., Guo, Y., Bour, S., Liu, D., et al. (2007). DAVID Knowledgebase: a gene-centered database integrating heterogeneous gene annotation resources to facilitate high-throughput gene functional analysis. BMC Bioinformat. 8:426. doi: 10.1186/1471-2105-8-426

Sherman, D. L., Wu, L. M., Grove, M., Gillespie, C. S., and Brophy, P. J. (2012). Drp2 and periaxin form Cajal bands with dystroglycan but have distinct roles in Schwann cell growth. J. Neurosci. 32, 9419-9428. doi: 10.1186/1471-21058-426

Tsutsumi, K., Yamashita, Y., Ushio, S., Kawashiri, T., Kaname, T., Fujita, S. et al. (2014). Oxaliplatin induces hypomyelination and reduced neuregulin 1 expression in the rat sciatic nerve. Neurosci. Res. 80, 86-90. doi: 10.1016/j.neures.2014.02.004

Verrier, J. D., Lau, P., Hudson, L., Murashov, A. K., Renne, R., and Notterpek, L. (2009). Peripheral myelin protein 22 is regulated post-transcriptionally by miRNA-29a. Glia 57, 1265-1279. doi: 10.1002/glia.20846

Vlachos, I. S., Kostoulas, N., Vergoulis, T., Georgakilas, G., Reczko, M., Maragkakis, M., et al. (2012). DIANA miRPath v.2.0: investigating the combinatorial effect of microRNAs in pathways. Nucleic Acids Res. 40, W498W504. doi: 10.1093/nar/gks494

Wong, M., and Martin, L. J. (2010). Skeletal muscle-restricted expression of human SOD1 causes motor neuron degeneration in transgenic mice. Hum. Mol. Genet. 19, 2284-2302. doi: 10.1093/hmg/ddq106

Zhou, F., Guan, Y., Chen, Y., Zhang, C., Yu, L., Gao, H., et al. (2013). miRNA-9 expression is upregulated in the spinal cord of ${ }^{\mathrm{G} 93 \mathrm{~A}}$-SOD1 transgenic mice. Int. J. Clin. Exp. Pathol. 6, 1826-1838.

Conflict of Interest Statement: The authors declare that the research was conducted in the absence of any commercial or financial relationships that could be construed as a potential conflict of interest.

Copyright (C) 2015 Dobrowolny, Bernardini, Martini, Baranzini, Barba and Musarò. This is an open-access article distributed under the terms of the Creative Commons Attribution License (CC BY). The use, distribution or reproduction in other forums is permitted, provided the original author(s) or licensor are credited and that the original publication in this journal is cited, in accordance with accepted academic practice. No use, distribution or reproduction is permitted which does not comply with these terms. 